\title{
Examining Thematic Variation in a Phenomenographical Study on Computational Physics
}

\author{
Nathaniel T. Hawkins, ${ }^{1}$ Michael J. Obsniuk, ${ }^{1,2}$ Paul W. Irving, ${ }^{1}$ and Marcos D. Caballero ${ }^{1,3,4}$ \\ ${ }^{1}$ Department of Physics and Astronomy, Michigan State University, East Lansing, MI, USA \\ ${ }^{2}$ Department of Physics, Kettering University, Flint, MI, USA \\ ${ }^{3}$ CREATE for STEM Institute, Michigan State University, East Lansing, MI, USA \\ ${ }^{4}$ Department of Physics and Center for Computing in Science Education, University of Oslo, Oslo, Norway
}

\begin{abstract}
Projects and Practices in Physics $\left(P^{3}\right)$ is a transformed, first-year introductory mechanics course offered at Michigan State University. The focus of the course is concept-based group learning implemented through solving analytic problems and computational modeling problems using the VPython programming environment. Interviews with students from $P^{3}$ were conducted to explore the variation of students' perceptions of the utility of solving computational physics problems in the classroom setting. A phenomenographic method is being used to develop categories of student experience with computational physics problems based on themes emerging across the different students' interviews. This paper will focus on exploring the variation within the theme of Computation Helps to Learn Physics that arose from our preliminary analysis of the data from a larger phenomenographic study. When examined on an individual basis, this theme provides important insights into students' perception of the use of computation, such as the way that students can engage with computation as a learning tool in a Physics classroom.
\end{abstract}

\section{INTRODUCTION}

Computation is an essential part of modern physics practice, as much of the physical world is now understood using computational modeling and analysis techniques. Helping students of physics learn computational methods, tools, and ways of thinking is likely to become a large part of future educational efforts [1]. However, the undergraduate environments in which students use computing are understudied $[2,3]$. This expanding field of research is open to many unanswered questions such as: "How do students learn computational tools and methods?", "How do students learn physics from engagement with computation?", and "How effective are students at appropriating and using computational practices in physics?"

The study presented here focuses on the development of the theme Computation Helps to Learn Physics, which is a description of the ways in which students' perceive the use of computation in their learning of physics. Students' perceptions of the tools and methods of instruction (e.g., computational activities) are important, as these perceptions can determine how a student chooses to engage in these activities and can thus affect how and what they learn [4]. This is particularly true when considering how negative views of computing can affect student learning [5]. In this paper, we present the first steps of a larger phenomenographic study that is aimed at developing categories of experience around students' perceptions of the utility of computing in their learning of physics. We present an emergent theme from the initial stages of our analysis that we feel is robust enough to provide important insights into how students are perceiving the interaction between computation and physics in our context.

\section{RESEARCH CONTEXT}

The students in Projects and Practices in Physics $\left(P^{3}\right)$ are primarily engineering students, with some physical sciences, computer science, and pre-professional majors as well. The structure of a $P^{3}$ semester is that students typically solve analytic problems in a group setting during one class period and computational problems in the subsequent class period. More details about $P^{3}$ are available in Irving et al. [6]. For the context of this study, we will be focusing on a suite of 3 problems students engage with over the first 3 weeks of the semester. The three computational problems in this suite are as follows: (1) a boat moving across a running river (vector addition and relative velocities), (2) a hovercraft accelerating off of a cliff and trying to land safely in the water below (2D kinematics), and (3) establishing the geosynchronous orbit of a satellite (Newtonian gravity). The students work on what we call minimally working programs, which are scripts that will run and produce a visual output in the VPython environment, but are missing the underlying physics equations needed to make a physically correct model. In this way, we can have the students focus on integrating physics into computation rather than learning a programming language. Twenty-one students were interviewed over the course of two semesters for this study. One-on-one interviews were conducted following the completion of all three computational problems. Interviews were conducted by one of the course instructors, one faculty member, one graduate student teaching assistant, and one undergraduate researcher.

\section{RESEARCH DESIGN AND ANALYSIS}

The larger scope of this study utilizes the framework of phenomenography, which can be summarized as the development of categories of description surrounding individuals' experiences with a certain phenomenon as a whole that is then used in facilitating the grasp of concrete cases of human understanding [7]. In utilizing this framework, we aim to develop categories of student perceptions of the utility of computation in an introductory mechanics course. The re- 


\begin{tabular}{clc} 
Label & Variation of Theme & N \\
\hline A & Computation Helps Me to Build a Conceptual Understanding of Physics & 7 \\
B & Engaging in the Practices of Computation Helps Me to Learn Physics & 14 \\
C & Computation Helps Me to Learn Physics When Interacting with Problems in Set Contexts & 5 \\
D & Computation Doesn't Help Me to Learn Physics, But Could Be Helpful to Others & 3 \\
E & Computation Does Not Help to Learn Physics in General & 4
\end{tabular}

TABLE I. Table summarizing the thematic variation present with the theme Computation Helps to Learn Physics. $N$ is a count of how many students (out of 21 total) exhibit this variation at some point during their interview. Note, some students exhibit multiple variations. Students may exhibit multiple varitaions throughout the course of the interview depending on the context in which they are describing their perceptions of computation.

sults and analysis presented in this paper are the first stages of the phenomenographic analysis process which is akin to qualitative research grounded in thematic analysis [8]. In semi-structured interviews students reflected on whether the computational activities they engaged in were helpful in their learning of physics. Analysis then focuses on reoccurring themes that emerge from the dataset with the expectation for phenomenography that several themes will emerge that are related to each other. Upon analysis of the transcribed dataset as a whole, there were many instances where similarities in the ways that students discussed computation as it relates to their learning of physics appeared. These were then reviewed to differentiate the modes of variation across this theme, which is discussed below. We acknowledge the analysis in this paper is not phenomenographic, as that would involve an investigation of their complete perceptions, but we believe this analysis at this stage can still offer valuable insights that can guide those who intend to integrate computation into their pedagogy. The larger study that this paper is a subset of will entail a full phenomenographic analysis.

\section{FINDINGS}

The theme discussed here is Computation Helps to Learn Physics. While the analysis of the dataset is still in a preliminary stage, exploring the variation within this theme is crucial as a standalone discussion because understanding students' perceptions of learning is important to the discussion of improving pedagogy [7, 9].

Table I shows a summary of the variations discussed in this section as well as a count of the number of students who display each variation in their interview data. It is important to note that students can display more than one variation of a theme during the course of their interview. As a result of this, variations within the theme are not mutually exclusive. In a phenomenographical study, we expect there to be multiple themes present across the dataset, each with its associated variation. Here, we are only looking at the presence of one theme, so individual instances of variations are counted as such, instead of being part of a larger result. The variations of the theme Computation Helps to Learn Physics are outlined below.

\section{A. Computation Helps Me to Build a Conceptual Understanding of Physics}

This variation can be identified in the student data in instances where the interviewees discussed experiences with the computational activities that helped them make a conceptual connection with physics. These instances are typically initiated by a student talking about their struggles with a particular physics concept going into a computational problem, but they then transition to a discussion about how the computational exercise helped them to understand the concept. One example that demonstrates this variation comes from Macku:

Macku: They were trying to explain that centrifugal force is not really an actual force. We just use it as a concept to explain things that are bigger. That was a big part, because we kept trying to say, 'oh, what kind of force are you going to need on it?' We were like, 'we only really need that force towards earth, we don't need the centrifugal force.' The centrifugal force wasn't in the code. I think it was not adding it to the code, but it was more understanding that we don't need it.

Macku mentions a specific example during her discussion of the geosynchronous orbit problem. The students use centrifugal force and equate that to the Newtonian gravitation equation to solve for the needed velocity to make a circular orbit. In coding the net force into the program, she noticed that it was not necessary to include the centrifugal force in her net force equation. Thus, for her it was a moment of realization that centrifugal force was not a physical force, but rather a method for performing certain approximations and calculations. Here, we can see a conceptual connection being made through the implementation of computation. The distinct characteristic of this theme is a direct mention to how engaging in computation helped the student to gain an understanding of a physics concept rather than a general statement about computation being helpful.

\section{B. Engaging in the Practice of Computation Helps Me to Learn Physics}

The next variation is where students refer to aspects of the computational program that can be summarized as computational practices. Students' descriptions often focused around practices nested in the overall practice of computational modeling like analyzing a visualization produced by VPython, or 
constructing the written lines of code that go into building the simulation. Students also perceive the act of iterating through the computational model itself and making decisions about variables as manifestations of the practice of "thinking like a Physicist." These are common aspects to programming in this setting $[10,11]$, and students identify how participating in these practices helps them to learn physics. This variation is demonstrated by Alan and Dota:

Alan: It allows me to model, model quickly, change quickly, and see the results of the changes to the model quickly. Because in these models, I can change specific variables and when I see the results, it allows me to draw some sort of visual picture of how I can manipulate real phenomenon with these variable manipulations.

Dota: Then we had to use friction to find acceleration, which we needed the old velocity for to find out how it changes, and so on. In the code, we would see equations call variables or reference values to do this same task. You're actually thinking like a Physicist would and applying what you're learning like this, which in turn helps me to learn.

The two examples show two different practices referenced by the students. Alan refers to the creation of the visual model and how changing specific variables in his code allows him to see the changes in his model, or as he says, allowing him to "manipulate real phenomenon." The ability to manipulate real phenomena through the practice of computational modeling is what Alan indicates is primarily how he learns physics. Dota references some work with using variables in different contexts and how in a program, she would see this unfold in instances where equations "call variables or reference values," which she associates with "thinking like a Physicist." This practice of thinking like a Physicist is what she identifies is the mechanism by which she learns. In these two discussions, these students indicate practices that each feels is helpful in his/her learning of physics. But the key distinction here is that by doing computation and engaging in the activity itself, it helps the student to learn physics.

\section{Computation Helps Me to Learn Physics When Interacting with Problems In Set Contexts}

This variation is seen in discussions where students describe some computational activities as helpful to their learning, while other problems as not beneficial. Based on statements made throughout interviews, students talk about it in ways that range from not getting additional information by solving a particular problem, not expanding upon their previous knowledge of the physics content, or not building a conceptual connection through that specific computational problem. Students identify some particular aspect or aspects of the computational problem as helping them to learn physics, but if those aspects were not present in the next problem or indicated to no longer be helpful, then the next problem would be viewed as not helping them to learn physics. Evidence for this can be seen in a statement made by Australia:

Australia: Sometimes I think [computation] helps and sometimes I think it doesn't help. The other [problems] helped me to grasp what happened when I changed something in the equation or added something to the equations. Rather, with this one I changed stuff and it didn't really do anything. It either made a dramatic difference or a slight one. I can picture a satellite moving around the earth, I didn't need the [code].

Australia is talking about the differences between the first two and the third of the computational problems. She talks about how the third problem, the geosynchronous orbit of a satellite, was something she could "picture" in her mind without the code. In the first two problems, changing some of the underlying equations in her program lead to a meaningful change in the visual output, which seems to be important to her learning of physics. The fact that the third problem either "made a dramatic difference or a slight one" establishes that the visualization aspect is important to her learning, but the key point to this variation is that the computational problems are only sometimes useful to her based on this criterion. This variation suggests that the value a student places on an aspect of computation in the current problem can depend on their experience with that aspect in previous problems.

\section{Computation Does Not Help Me to Learn Physics, But Could Be Helpful to Others}

There arose instances in the interviews where students would discuss how the computational portions of the problems were not helpful to their learning. This could be based on several factors, such as prior experience/knowledge with computation or physics or the lack thereof. What sets this apart from other variations is that these students then continue to talk about how it should or would help someone else to learn. This is evident in the following quote from Captain:

Captain: Two people in my group haven't taken physics, so I think that helps a lot of them understand what they're doing. They actually see a model of what they [coded]. It helps a lot of the understanding what you did, when you actually get to see what you did. I would say no in my case, but it should have helped someone else. Most of the things we are seeing in physics now I already learned. So far it's more like getting better at physics, not so much at learning physics for me. I would say what I did so far is something I already did in high school.

Captain mainly focuses his discussion on the fact that this is mostly material covered in his high school physics class, and thus he is not learning any new material. He does mention "getting better at physics," but this idea was not further explored. What is of interest in his discussion is that he talks about two of his group members who had not taken physics previously. Being able to "model" what they are coding should, as he says, help a lot with understanding what they had done by actually "seeing what they did." This idea of "seeing" could refer to the visualization produced by VPython or the written-out lines of code. In other cases, students have said that the computational problems were useful to other students who had some prior computational experience, but not to them because they had never coded before. 


\section{E. Computation Does Not Help to Learn Physics In General}

Different from the variation above, students here talk about how they do not believe that computation helps to learn physics in general. Instead of being unhelpful only to the individual's learning of physics or not helpful in solving only a few specific problems, they consider it to not be helpful in any case. Some evidence to demonstrate this variation is given by Vandermeer:

Vandermeer: I don't really learn the physics. It takes away from the physics when I'm still trying to figure out how to do an exponent, like two asterisks, parentheses, little things like that. I feel like having a programming class as pre-req would be quite helpful. I think the [analytic] labs are better at that. I enjoy pen and paper just because I don't have any coding experience.

Vandermeer's discussion revolves around the idea that computation isn't helpful for learning physics based on her experiences, because to her, the programming "takes away from the physics." The reasoning she provides is that she doesn't have any coding experience and the syntax is difficult enough for her that her focus is shifted from the physics content to writing a working program. She indicates that the analytic problems are better at teaching her the material.

\section{DISCUSSION AND CONCLUSIONS}

We presented five major variations present in this emergent theme. Three of the variations point towards a positive impact on student learning via computation and students having some positive computational experience in the class (A, B, \& C), while two are associated with students not viewing their computational experiences as having a positive impact on their learning (D \& E). Only four students provided discussions that identify computation as not being helpful to learning physics. However, the variation in their reasoning provides a platform to address their concerns through alterations to the design of activities and messaging in the class- room.

Delving into the quantitative counts, the variation with the largest number was variation B. Variation B's focus on engaging in the practices of computation and the perception that "thinking like a physicist" is working with computational models demonstrates a positive alignment with the learning environment's design focus on practices and the instructor's meta-messaging around the role of computation in physics. We can also see from Table I that seven students displayed variation $\mathrm{A}$ at some point during their interview, which is a positive indicator that the computational activities are playing a role in the development of student understanding in $P^{3}$. A deeper investigation into the specific aspects of computational activities that supported the development of understanding could again support future curriculum design.

From a methodology perspective, these variations include some deeper nuances that we expect to understand further when combined with other emergent themes during the complete phenomenographic analysis. For instance, several of the themes explored in the larger study focus on the role of the visualizations produced by the computational models in students' learning. A future theme or variation that may emerge in the complete analysis of our dataset could be focused on student use of visualization. This could then connect to the theme presented in this paper in evidence such as the quote displayed by Captain in Variation D. This student references the utility of "seeing" in learning physics. These connnections between themes will help fill out our outcome space of students' perceptions of the utility of computation where multiple themes are used to reinforce one another [9].

Continued analysis of this dataset will yield additional supporting information to strengthen the variations within this theme and a greater understanding of the effects an integrated computational approach to learning physics can have on students. Due to the relatively short time period over which this study occurs, our future work will also focus on understanding how student perceptions of utility of computation change over longer periods of time.
[1] AAPT Undergraduate Curriculum Task Force. "AAPT Recommendations for Computational Physics in the Undergraduate Physics Curriculum." 2016.

[2] Aiken, J. et al. "Understanding student computational thinking with computational modeling." Physics Education Research Conference. Volume 1513. 2012.

[3] Caballero, M., Kohlmyer, M., Schatz, M. "Implementing and assessing computational modeling in introductory mechanics." Physical Review Physics Education Research. Volume 8, Issue 2. 2012.

[4] Landau, R. "Computational Physics: A Better Model for Physics Education." IEEE Computer Society and American Institute of Physics. 2006

[5] Tsai, M., Chin-Chung T. "Student computer achievement, attitude, and anxiety: The role of learning strategies." Journal of Educational Computing Research 28.1, 2003.
[6] Irving, P., Obsniuk M., Caballero, D. " $P^{3}$ : a practice focused learning environment." European Journal of Physics. Volume 38, No. 5. 2017.

[7] Marton, F. "Phenomenography-Describing conceptions of the world around us." Instructional Science. Volume 2. July, 1981.

[8] Braun, V. and Clarke, V. (2006) Using thematic analysis in psychology. Qualitative Research in Psychology. Volume 3 (2).

[9] Svensson, L. "Theoretical Foundations of Phenomenography." Higher Education Research \& Development. Volume 16, No. 2. 1997.

[10] Backer, A. "Computational Physics Education with Python." IEEE Computer Society and American Institute of Physics. 2007.

[11] Esquembre, F. "Computers in physics education." Computer Physics Communications. No. 147. 2002. 